\title{
ON COMPOSITIONS OF CONFORMAL IMMERSIONS
}

\author{
MARCOS DAJCZER AND ENALDO VERGASTA
}

(Communicated by Jonathan M. Rosenberg)

\begin{abstract}
We consider conformal immersions of a manifold $M^{n}, n \geq 6$, into conformally flat manifolds. If the principal curvatures of $f: M^{n} \rightarrow N_{c f}^{n+1}$ have multiplicities at most $n-4$, we show that any $g: M^{n} \rightarrow \tilde{N}_{c f}^{n+2}$ can locally be written as $g=\rho \circ f$, where $\rho: N_{c f}^{n+1} \rightarrow \tilde{N}_{c f}^{n+2}$ is a conformal immersion.
\end{abstract}

\section{INTRODUCTION}

A classical result due to Cartan [Ca] states that a codimension one conformal immersion $f: M^{n} \rightarrow N_{c f}^{n+1}$ of an $n$-dimensional Riemannian manifold into a conformally flat Riemannian manifold is (locally) conformally rigid if $n \geq 5$ and the maximal multiplicity of the principal curvatures satisfies $\nu_{f}^{c} \leq n-3$ everywhere. Then any other conformal immersion $g: M^{n} \rightarrow \widetilde{N}_{c f}^{n+1}$ is locally a composition $g=\rho \circ f$ for some local conformal diffeomorphism $\rho: N_{c f}^{n+1} \rightarrow$ $\tilde{N}_{c f}^{n+1}$. Cartan's result was extended to codimension greater than one in [dCD].

For fixed $k \geq 2$, a natural problem is to find conditions on $f: M^{n} \rightarrow N_{c f}^{n+1}$ which imply that any conformal immersion $g: M^{n} \rightarrow \widetilde{N}_{c f}^{n+k}$ is locally a conformal composition. That $g$ is a local conformal composition means that, for each point $x \in M^{n}$, there exists a neighborhood $V \subset M$ of $x$ and a conformal immersion $\rho: W \subset N_{c f}^{n+1} \rightarrow \widetilde{N}_{c f}^{n+k}$ of an open subset of $N_{c f}^{n+1}$ containing $f(V)$ such that $g=\rho \circ f$ along $V$. When $k=2$, we prove the following result.

Theorem 1. Let $f: M^{n} \rightarrow N_{c f}^{n+1}$ be a conformal immersion. Assume that $n \geq 6$ and $\nu_{f}^{c}(x) \leq n-4$ everywhere. If $g: M^{n} \rightarrow \widetilde{N}_{c f}^{n+2}$ is a conformal immersion then there exists an open dense subset $\mathscr{U} \subset M$ such that, when restricted to $\mathscr{U}$, $g$ is a local conformal composition.

\section{THE PROOF}

We say that a submanifold $\bar{N}^{n+1} \subset \widetilde{N}_{c f}^{n+2}$ is a conformally flat hypersurface if, with the metric induced by the inclusion map, $\bar{N}^{n+1}$ is conformally flat. Using Cartan's result, it is easy to check that Theorem 1 is equivalent to the following:

Received by the editors August 12, 1991.

1991 Mathematics Subject Classification. Primary 53C42; Secondary 53A30. 
Theorem 2. Let $f: M^{n} \rightarrow N_{c f}^{n+1}$ be a conformal immersion. Assume that $n \geq 6$ and $\nu_{f}^{c}(x) \leq n-4$ everywhere. If $g: M^{n} \rightarrow \widetilde{N}_{c f}^{n+2}$ is a conformal immersion then there exists an open dense subset $\mathscr{U} \subset M^{n}$ such that $\left.g\right|_{\mathscr{U}}$ is locally contained in a conformally flat hypersurface of $\tilde{N}_{c f}^{n+2}$.

To prove Theorem 2 we will make use of the following lemma on flat bilinear forms. We refer the reader to [dCD] or [Da] for notation, definitions, and some basic facts.

Lemma 3. Let $\beta: V \times V \rightarrow W^{k, 2}, k \geq 3$, be a nonzero symmetric bilinear form. Assume that $\beta$ is flat and $\operatorname{dim} N(\beta)<\operatorname{dim} V-\operatorname{dim} W$. Then $W$ admits an orthogonal direct sum decomposition $W=W_{1}^{r, r} \oplus W_{2}^{k-r, 2-r}$, where $r=1$ or 2 , such that if $\beta_{1}$ and $\beta_{2}$ are the $W_{1}$ and $W_{2}$ components of $\beta$, respectively, then

(i) $\beta_{1}$ is nonzero and null,

(ii) $\beta_{2}$ is flat and $\operatorname{dim} N\left(\beta_{2}\right) \geq \operatorname{dim} V-\operatorname{dim} W_{2}$.

Proof. Analogous to that of Lemma 2.2 in [dCD].

Proof of Theorem 2. We may assume that $N_{c f}^{n+1}=S^{n+1}$ is the unit Euclidean sphere, that $\tilde{N}_{c f}^{n+2}=\mathbf{R}^{n+2}$ is the flat Euclidean space, and that $M^{n}$ is endowed with the metric induced by $g$. We consider $S^{n+1}$ isometrically embedded in the light-cone $\mathbf{V}^{n+2}$ of the flat Lorentzian space $\mathbf{L}^{n+3}$ and contained in an $(n+2)$-dimensional affine hyperplane orthogonal to the axis of $\mathbf{V}^{n+2}$.

The map $F: M^{n} \rightarrow \mathbf{V}^{n+2} \subset \mathbf{L}^{n+3}$ defined by

$$
F(x)=\frac{1}{\varphi(x)} f(x)
$$

is an isometric immersion, where $\varphi: M^{n} \rightarrow \mathbf{R}$ is the positive function satisfying

$$
\left\langle f_{*}(x) X, f_{*}(x) Y\right\rangle=\varphi^{2}(x)\langle X, Y\rangle
$$

for any $X, Y \in T_{x} M$.

As in [dCD] or [Da], for a fixed point $x \in M^{n}$, the vector-valued second fundamental form $\alpha_{F}: T M \times T M \rightarrow T_{F} M^{\perp}$ of $F$ in $\mathbf{L}^{n+3}$ is given by

$$
\alpha_{F}=\left(\left\langle\alpha_{F}, \eta\right\rangle+\langle,\rangle\right) \xi+\left\langle\alpha_{F}, \eta\right\rangle \eta+\alpha_{F}^{*},
$$

where the basis $\xi, \eta$ for the orthogonal complement of $T_{f(x)} M^{\perp}$ into $T_{F(x)} M^{\perp}$ verifies

$$
\langle\xi, \xi\rangle=1, \quad\langle\xi, \eta\rangle=0, \quad\langle\eta, \eta\rangle=-1
$$

and $F(x)=\xi+\eta$. Here $\alpha_{F}^{*}$ is the $T_{f(x)} M^{\perp}$ component of $\alpha_{F}$ and satisfies

$$
\alpha_{F}^{*}=\alpha_{f} / \varphi \text {. }
$$

Now let

$$
W=T_{g(x)} M^{\perp} \oplus \operatorname{Span}\{\xi\} \oplus \operatorname{Span}\{\eta\} \oplus T_{f(x)} M^{\perp}
$$

be given the natural metric $\langle(\rangle$,$\rangle of type (3,2)$. Define $\beta: T_{x} M \times T_{x} M \rightarrow W$ by

$$
\beta=\alpha_{g} \oplus\left(\left\langle\alpha_{F}, \eta\right\rangle+\langle,\rangle\right) \xi \oplus\left\langle\alpha_{F}, \eta\right\rangle \eta \oplus \alpha_{F}^{*} .
$$


A straightforward computation shows that

$$
\begin{aligned}
&\langle\langle\beta(X, Y), \beta(Z, W)\rangle\rangle-\langle\langle\beta(X, W), \beta(Z, Y)\rangle\rangle \\
&=\left\langle\alpha_{g}(X, Y), \alpha_{g}(Z, W)\right\rangle-\left\langle\alpha_{g}(X, W), \alpha_{g}(Z, Y)\right\rangle \\
&-\left\langle\alpha_{F}(X, Y), \alpha_{F}(Z, W)\right\rangle+\left\langle\alpha_{F}(X, W), \alpha_{F}(Z, Y)\right\rangle,
\end{aligned}
$$

and the Gauss equations for $g$ and $F$ imply that $\beta$ is flat.

By definition of $\beta$, we have $\beta(X, X) \neq 0$ for $X \neq 0$; thus, $N(\beta)=0$. By Lemma $3, W=W_{1} \oplus W_{2}$ decomposes orthogonally so that $\beta=\beta_{1} \oplus \beta_{2}$, where

$$
\beta_{1}: T_{x} M \times T_{x} M \rightarrow W_{1}^{r, r}, \quad r \in\{1,2\},
$$

is nonzero and null and

$$
\beta_{2}: T_{x} M \times T_{x} M \rightarrow W_{2}^{3-r, 2-r}
$$

is flat satisfying $\operatorname{dim} N\left(\beta_{2}\right) \geq n-5+2 r$.

We claim that $r=2$. Assume $r=1$. It follows that $\beta_{1}=\phi \gamma$, where $\gamma \in W_{1}$ is a null vector and $\phi$ is a real-valued symmetric bilinear form. Thus there exists a unit vector $\delta \in T_{g(x)} M^{\perp}$ such that

$$
\gamma=\cos \theta \delta+\sin \theta \xi+\cos \bar{\theta} \eta+\sin \bar{\theta} N,
$$

where $N \in T_{f(x)} M^{\perp}$ is a unit vector. By definition, we have $Z \in N\left(\beta_{2}\right)$ if and only if $\beta(Z, X)=\beta_{1}(Z, X)=\phi(Z, X) \gamma$ for all $X \in T_{X} M$; therefore,

$$
\begin{gathered}
\left\langle\alpha_{F}(Z, X), \eta\right\rangle+\langle Z, X\rangle=\phi(Z, X) \sin \theta, \\
\left\langle\alpha_{F}(Z, X), \eta\right\rangle=\phi(Z, X) \cos \bar{\theta}
\end{gathered}
$$

and

$$
\left\langle\alpha_{F}^{*}(Z, X), N\right\rangle=\phi(Z, X) \sin \bar{\theta}
$$

for all $Z \in N\left(\beta_{2}\right)$ and $X \in T_{x} M$. From (2) and (3) we get

$$
\phi(Z, X)(\sin \theta-\cos \bar{\theta})=\langle Z, X\rangle,
$$

which implies $\sin \theta-\cos \bar{\theta} \neq 0$. From (4) and (5) we obtain

$$
\left\langle\alpha_{F}^{*}(Z, X), N\right\rangle=\frac{\sin \bar{\theta}}{\sin \theta-\cos \bar{\theta}}\langle Z, X\rangle .
$$

Using (1), we conclude from (6) that $f$ has a principal curvature with multiplicity at least $\operatorname{dim} N\left(\beta_{2}\right) \geq n-3$. This is a contradiction and proves the claim.

Since $r=2$, we have $\beta_{1}=\phi_{1} \gamma_{1}+\phi_{2} \gamma_{2}$, where $\phi_{1}, \phi_{2}$ are real-valued symmetric bilinear forms and $\gamma_{1}, \gamma_{2}$ are orthogonal null vectors. So we may write

$$
\gamma_{1}=\eta+\cos u \xi+\sin u \delta_{1}
$$

and

$$
\gamma_{2}=N+\cos v \xi+\sin v \delta_{2},
$$

where $\delta_{1}, \delta_{2}$ are unit vectors in $T_{g(x)} M^{\perp}$ verifying

$$
\cos u \cos v+\sin u \sin v\left\langle\delta_{1}, \delta_{2}\right\rangle=0 \text {. }
$$


Clearly, $\phi_{1}=\left\langle\alpha_{F}, \eta\right\rangle$ and $\phi_{2}=\left\langle\alpha_{F}^{*}, N\right\rangle$. Hence,

$$
\beta_{1}=\left\langle\alpha_{F}, \eta\right\rangle\left(\eta+\cos u \xi+\sin u \delta_{1}\right)+\left\langle\alpha_{F}^{*}, N\right\rangle\left(N+\cos v \xi+\sin v \delta_{2}\right) .
$$

For any $Z \in N\left(\beta_{2}\right)$ and $X \in T_{x} M, \beta(Z, X)=\beta_{1}(Z, X)$ is equivalent to

$$
\alpha_{g}(Z, X)=\left\langle\alpha_{F}(Z, X), \eta\right\rangle \sin u \delta_{1}+\left\langle\alpha_{F}^{*}(Z, X), N\right\rangle \sin v \delta_{2}
$$

and

$$
\left\langle\alpha_{F}(Z, X), \eta\right\rangle(1-\cos u)+\langle Z, X\rangle=\left\langle\alpha_{F}^{*}(Z, X), N\right\rangle \cos v .
$$

Thus, from $\nu_{f}^{c} \leq n-4$, we have $1-\cos u \neq 0$ and $\cos v \neq 0$; therefore,

$$
\alpha_{g}(Z, X)=\left\langle\alpha_{F}(Z, X), \eta\right\rangle\left(\sin u \delta_{1}+\operatorname{tg} v(1-\cos u) \delta_{2}\right)+\operatorname{tg} v\langle Z, X\rangle \delta_{2}
$$

and

$$
\begin{aligned}
\alpha_{g}(Z, X)= & \left\langle\alpha_{F}^{*}(Z, X), N\right\rangle\left(\frac{\sin u \cos v}{1-\cos u} \delta_{1}+\sin v \delta_{2}\right) \\
& -\frac{\sin u}{1-\cos u}\langle Z, X\rangle \delta_{1} .
\end{aligned}
$$

We easily conclude from (10) that $g$ has a normal direction $\sigma$ such that the tangent-valued second fundamental form $A_{\sigma}$ in this direction has an eigenvalue with multiplicity at least $n-1$ whose eigenspace contains $N\left(\beta_{2}\right)$.

From $r=2$ we have that $\operatorname{dim} S(\beta)=2,3$. We claim that $\operatorname{dim} S(\beta)=2$ if and only if $\sigma$ is an umbilical direction. First observe that $\operatorname{dim} S(\beta)=2$ if and only if $\beta_{2}=0$, and if $\beta_{2}=0$ then $\sigma$ is umbilical by equation (10). Conversely, if $A_{\sigma}=c I$, consider the vector $\zeta=\sigma / c-\xi-\eta$. Then $\zeta$ is not null and $\langle\langle\beta, \zeta\rangle\rangle=0$. This implies that $\operatorname{dim} S(\beta)=2$ and proves the claim.

Assume that $\operatorname{dim} S(\beta)=3$ on an open subset $V \subset M^{n}$. The 2-dimensional distribution $S(\beta) \cap S(\beta)^{\perp}$ is the (maximal) degeneracy space of the restriction of $\langle\langle\rangle$,$\rangle to the smooth distribution S(\beta)$ and, therefore, is smooth. It follows easily that the vector fields $\delta_{1}, \delta_{2}$ and the functions $u, v$ in (7) and (8) can be taken to be smooth on $V$. The same conclusion holds on any open subset of $M$ where $\operatorname{dim} S(\beta)=2$.

Let $\mathscr{W} \subset M$ be the open subset of points where $\operatorname{dim} S(\beta)=3$, and let $\mathscr{U}_{1}$ be the interior of $M \backslash \mathscr{W}$. Let $\sigma$ be a smooth umbilical unit normal vector field defined on a connected component $U_{\lambda}$ of $\mathscr{U}_{1}$. We claim that $\sigma$ is parallel with respect to the normal connection of $g$. In fact, if $\sigma$ is not parallel at $x \in U_{\lambda}$, we easily conclude from the Codazzi equation for $A_{\sigma}$ that the second fundamental form $A_{\sigma^{\perp}}$ has a principal curvature with multiplicity at least $n-1$. The same holds in a neighborhood $W \subset U_{\lambda}$ of $x$, and it is a well-known fact that $W$ must be conformally flat (cf. [CY]). By the classical Cartan-Schouten theorem for conformally flat hypersurfaces, we conclude that $\nu_{f}^{c} \geq n-1$ on $W$, which is a contradiction and proves the claim. It follows from the claim that $g\left(U_{\lambda}\right)$ is contained in an umbilical hypersurface of $\mathbf{R}^{n+2}$.

For a connected component $V_{\lambda}$ of $\mathscr{W}$, let $\sigma$ be a smooth unit normal vector field such that the second fundamental form $A_{\sigma}$ has eigenvalues $\mu, \lambda$ with multiplicities 1 and $(n-1)$, respectively. Set $\Delta=\operatorname{ker}\left(A_{\sigma}-\lambda I\right)$. We claim that $\lambda$ is constant and $\sigma$ is parallel along $\Delta$. Consider orthonormal vector fields $Y_{1}, \ldots, Y_{n-1} \in \Delta$ such that $\nabla_{Y_{j}}^{\frac{1}{j}} \sigma=0$ for $2 \leq j \leq n-1$. From the 
Codazzi equation for $A_{\sigma}, Y_{i}$, and $Y_{j}$ for $2 \leq i \neq j \leq n-1$, we easily conclude that

$$
Y_{j}(\lambda)=0, \quad 2 \leq j \leq n-2 .
$$

Now the Codazzi equation for $A_{\sigma}, Y_{1}$, and $Y_{j}$ yields

$$
\left\langle\nabla_{Y_{1}}^{\perp} \sigma, \sigma^{\perp}\right\rangle\left\langle A_{\sigma^{\perp}} Y_{i}, Y_{j}\right\rangle=0, \quad 1 \leq i \neq j \leq n-1
$$

and

$$
Y_{1}(\lambda)=\left\langle\nabla_{Y_{1}}^{\perp} \sigma, \sigma^{\perp}\right\rangle\left\langle A_{\sigma^{\perp}} Y_{j}, Y_{j}\right\rangle, \quad 2 \leq j \leq n-1 .
$$

If at some point $\left\langle\nabla_{Y_{1}}^{\perp} \sigma, \sigma^{\perp}\right\rangle \neq 0$, we obtain from (12) and (13) that $\operatorname{Span}\left\{Y_{2}, \ldots, Y_{n-1}\right\}$ contains an $(n-3)$-dimensional umbilical subspace for $g$. Now (1) and (11) imply that $\nu_{f}^{c}(x) \geq n-3$, which is not possible, and this proves the claim.

Set $\mathscr{U}=\mathscr{U}_{1} \cup \mathscr{U}_{2} \cup \mathscr{U}_{3}$, where $\mathscr{U}_{3} \subset \mathscr{W}$ is the open subset where $\lambda \neq 0$ and $\mathscr{U}_{2}$ is the interior of $\mathscr{W} \backslash \mathscr{U}_{3}$.

The image under $g$ of any connected component of $\mathscr{U}_{2}$ is contained in a flat hypersurface of $\mathbf{R}^{n+2}$ by Proposition 3 of [DG]. If $V_{\lambda}$ is a connected component of $\mathscr{U}_{3}$, define $c: V_{\lambda} \rightarrow \mathbf{R}^{3}$ by

$$
c(x)=g(x)+r(x) \sigma(x), \quad r(x)=1 / \lambda(x) .
$$

For all $Y \in \Delta$, we have

$$
\widetilde{\nabla}_{Y} c=Y-r A_{\sigma} Y=0
$$

where $\widetilde{\nabla}$ denotes the canonical connection of $\mathbf{R}^{n+2}$. If $X$ is a unit tangent vector field orthogonal to $\Delta$, we get

$$
\widetilde{\nabla}_{X} c=X-X(r) \sigma-r A_{\sigma} X-r \nabla_{X}^{\perp} \sigma .
$$

In particular, since $\sigma$ is not an umbilical direction, we have

$$
\left\|\widetilde{\nabla}_{X} c\right\|^{2}>|X(r)|^{2}
$$

hence, from the curve $c$ and the function $r$ we can construct a conformally flat hypersurface in $\mathbf{R}^{n+2}$ as described in [dCDM] or [Da] which contains $g\left(V_{\lambda}\right)$. This concludes the proof.

\section{REFERENCES}

[Ca] E. Cartan, La déformation des hypersurfaces dans l'espace conforme réel a $n \geq 5$ dimensions, Bull. Soc. Math. France 45 (1917), 57-121.

[dCD] M. do Carmo and M. Dajczer, Conformal rigidity, Amer. J. Math. 109 (1987), 963-985.

[CY] B. Y. Chen and K. Yano, Umbilical submanifolds with respect to a nonparallel normal direction, J. Differential Geom. 8 (1973), 589-597.

[dCDM] M. do Carmo, M. Dajczer, and F. Mercuri, Compact conformally flat hypersurfaces, Trans. Amer. Math. Soc. 288 (1985), 189-203.

[Da] M. Dajczer et al., Submanifolds and isometric immersions, Math. Lecture Series, vol. 13, Publish or Perish, Houston, TX, 1990.

[DG] M. Dajczer and D. Gromoll, Isometric deformations of compact Euclidean submanifolds in codimension 2, Duke Math. J. (to appear).

IMPA, Est. Dona Castorina 110, 22460-320 Rio de JANeiro, Brazil

E-mail address: MARCOS@IMPA.BR

Institute of Mathematics, Universidade federal da Bahia, Av. Ademar de Barros s/N, 40210 Salvador, BRAZIL 